\title{
Influência da preocupação com quedas na mobilidade e na força de reação do solo em idosas durante descida de escada
}

\author{
Influence of concern about falls in mobility and ground reaction force \\ in elderly women's during stairs descent
}

\author{
Danilo de Oliveira Silva ${ }^{1}$, Marcella Ferraz Pazzinatto², Maíra Caroline de Oliveira ${ }^{3}$, Fernando Amâncio Aragão ${ }^{4}$, \\ Carlos Eduardo de Albuquerque ${ }^{5}$ \\ ${ }^{1}$ Mestre em Fisioterapia, Faculdade de Ciências e tecnologia da Universidade Estadual Paulista Júlio de Mesquita Filho (FCT-UNESP). \\ ${ }^{2}$ Mestranda em Fisioterapia, FCT-UNESP. \\ ${ }^{3}$ Fisioterapeuta, Universidade Estadual do Oeste do Paraná (UNIOESTE). \\ ${ }^{4}$ Doutor em Ciências do Movimento Humano pela Universidade Federal do Rio Grande do Sul (UFRGS). Professor adjunto da UNIOESTE. \\ ${ }^{5}$ Mestre em Bioengenharia pela Universidade do Vale do Paraíba (UNIVAP). Professor adjunto da UNIOESTE.
}

\section{RESUMO}

Objetivos: Verificar a diferença entre idosas com e sem preocupação com quedas na mobilidade e na força vertical de reação do solo durante a descida de escada.

Métodos: O estudo incluiu idosas atendidas pelo Centro de Reabilitação Física da Universidade Estadual do Oeste do Paraná com idade entre 60 e 75 anos, sem doenças neurológicas e cardiovasculares que acarretassem risco pelas atividades do estudo. Foram excluídas também idosas com órteses para locomoção, doenças osteoarticulares ou cirurgias de membros inferiores e histórico de queda. As participantes foram divididas em dois grupos, com e sem preocupação com quedas em atividades diárias, identificados pela Falls Efficacy Scale International traduzida para o português. Para avaliação da mobilidade, todas as voluntárias realizaram o teste Timed Up and Go. A força de reação do solo foi avaliada em cinco descidas de escada para coleta dos dados cinéticos (primeiro pico da força vertical de reação do solo) através de uma plataforma de força alocada no quarto degrau de uma escada de sete degraus. Após ser confirmada a normalidade dos dados, o teste $t$ de Student para amostras independentes foi utilizado para comparação entre os grupos.

Resultados: A amostra foi composta por dezessete idosas sem preocupação com quedas (idade de $66,57 \pm 4,1$ anos) e dezesseis idosas com preocupação com quedas (idade de 66,67 $\pm 5,8$ anos). O grupo sem preocupação com quedas desempenhou o teste Timed Up and Go de forma mais veloz $(9,71 \pm 1,02$ segundos) comparado ao grupo preocupação com quedas $(11,5 \pm 1,04$ segundos $)(p=0,008)$. Não houve diferença entre os grupos para o primeiro pico da força de reação do solo vertical $(\mathrm{p}=0,66)$.

Conclusões: Idosas com preocupação com queda apresentaram menor desempenho no teste funcional do que idosas com ausência dessa preocupação. Entretanto, não houve diferença entre os grupos quanto à variável cinética.

DESCRITORES: IDOSO; CINÉTICA; LOCOMOÇÃO; MEDO; QUESTIONÁRIOS.

\section{ABSTRACT}

Aims: To investigate the difference between elderly women with and without concern about falls in mobility and vertical ground reaction force during stair descent.

Methods: The study included older women assisted in the Centre for Physical Rehabilitation of the Universidade Estadual do Oeste do Paraná, aged between 60 and 75 years, without neurological and cardiovascular disease involving risk by the activities of the study. Women with orthoses for locomotion, osteoarticular diseases or surgery of lower limbs, and previous fall history were also excluded. The participants were divided into two groups, with and without concern about falls in daily activities, identified by the Falls Efficacy Scale International translated into Portuguese. To assess mobility, all subjects performed the Timed Up and Go Test. Ground reaction force was assessed by walking steps down five times to collect kinetic data (first peak of the vertical ground reaction force) through a force platform allocated in the fourth step of a staircase of seven steps. After confirmed normality of data, Student $t$ test for independent samples was used to compare the groups.

Results: The sample was composed of seventeen elderly women with no concern about falling (aged $66.57 \pm 4.1$ years) and sixteen elderly women with concerns about falling (aged $66.67 \pm 5.8$ years). The group without concern with falls performed the Timed Up and Go Test faster $(9.71 \pm 1,02$ seconds) than the group with concern about falls $(11.5 \pm 1,04$ seconds $)(p=0.008)$. There was no difference between groups on the first peak of vertical ground reaction force $(\mathrm{p}=0.66)$.

Conclusions: Elderly women with concern about falls had lower performance in the functional test than elderly women with no such concern. However, there was no difference between the groups in terms of the kinetic variable.

KEY WORDS: ELDERLY; KINETICS; LOCOMOTION; FEAR; QUESTIONNAIRES 


\section{INTRODUÇÃO}

A transição demográfica encontra-se em diferentes fases ao redor do mundo, tendo certamente como resultado o envelhecimento populacional. ${ }^{1}$ Este fenômeno exige maiores cuidados com a população idosa, devido às disfunções que acometem os últimos anos de suas vidas. ${ }^{1}$ Igualmente, a proporção de pessoas idosas cresce em ritmo maior do que o número de pessoas que nascem. ${ }^{2}$

Dentre a série de alterações morfofuncionais que ocorrem durante o processo de envelhecimento, pode ser destacado o declínio do sistema musculoesquelético, o qual apresenta diminuição tanto na força quanto na massa muscular. ${ }^{3,4}$ Por consequência, os indivíduos tornam-se mais frágeis e apresentam maior risco de sofrer acidentes, o que pode gerar dificuldades ou até impossibilitar a realização das atividades de vida diária de forma independente..$^{5,6}$

Um dos grandes desafios encontrados pelos idosos durante a locomoção é a transposição de pisos, sendo a subida/descida de escadas relatada como uma entre as cinco tarefas mais difíceis desempenhadas nessa faixa etária. ${ }^{7}$ Segundo a revisão de Startzell et al., ${ }^{5}$ em torno de $10 \%$ das mortes relacionadas com quedas ocorreram durante essas atividades. E cerca de $80 \%$ das quedas ocorrem no momento da descida de escadas. Este fato demonstra a importância de investigar esta atividade funcional na qual o risco de cair está muito presente. ${ }^{8}$ Com o aumento significativo do risco de quedas ao avançar da idade, a preocupação com queda também se encontra presente em $30 \%$ da população acima de 60 anos independente do histórico de quedas. ${ }^{9}$ Mulheres apresentam quatro vezes mais preocupação do que homens. ${ }^{10}$ Essa porcentagem é duplicada naqueles idosos com pelo menos um episódio anterior de queda. ${ }^{11}$ A preocupação afeta os idosos de maneira geral, no entanto, idosos que manifestam o sentimento de receio têm maiores possibilidades de queda do que os demais. ${ }^{2}$ Os testes clínicos, como o Timed Up and Go (TUG), ${ }^{12,13}$ são importantes indicadores de função dos idosos, inferindo riscos de queda e independência funcional. ${ }^{14}$ No entanto, existe carência de estudos que associem a preocupação de cair quantificada em escalas, com testes e atividades funcionais. A utilização de testes funcionais e a aplicação de questionários validados são perfeitamente viáveis na prática clínica. Além de serem ferramentas de baixo custo, são capazes de retratar fielmente situações cotidianas. Pesquisas que associem a preocupação em cair com resultados de testes clínicos são de fundamental importância, pois abrem-se oportunidades para o terapeuta avaliar o idoso de forma multidisciplinar, e não apenas considerando os fatores musculoesqueléticos decorrentes do envelhecimento.

A avaliação das características biomecânicas de idosos em diferentes tarefas cotidianas, mais simples como sentar e levantar, ou mais complexas como a descida de escadas, vem ganhando espaço na literatura, com intuito de refinar protocolos e possibilitar que futuras intervenções tenham ação diretamente na causa do problema. A análise cinética através de plataforma de força pode trazer informações interessantes quanto às forças de reação do solo nessa população, identificando se há sobrecarga nos membros inferiores. A partir dessas informações é possível definir intervenções específicas para uma determinada população. O receio da queda pode levar a alterações desse parâmetro, o que alteraria a biomecânica normal e poderia gerar predisposição das articulações dos membros inferiores a lesões. ${ }^{15-18}$

Desta forma, é fundamental analisar as características biomecânicas durante a atividade de descer escadas e os testes funcionais, comparando os idosos que têm receio de cair com aqueles sem essa preocupação. Essa análise possibilita conhecer e proporcionar futuras estratégias preventivas, mantendo o idoso independente no quesito mobilidade. O presente estudo teve como objetivo verificar a diferença entre idosas com e sem preocupação com quedas na mobilidade e na força vertical de reação do solo durante a descida de escada.

\section{MÉTODOS}

\section{Caracterização da amostra}

Participaram do estudo idosas atendidas pelo Centro de Reabilitação Física (CRF) da Universidade Estadual do Oeste do Paraná (UNIOESTE), que atenderam ao recrutamento feito por meio de anúncios em academias de terceira idade, postos de saúde, clínicas de reabilitação física e parques da cidade de Cascavel, Paraná. Para serem incluídas no estudo as voluntárias deveriam ter idade entre 60 e 75 anos e não apresentar doenças neurológicas e cardiovasculares que as colocassem em risco durante as atividades propostas no estudo. Também não foram incluídas voluntárias com órteses para locomoção, com relato de doenças osteoarticulares de membros inferiores ou de cirurgias de qualquer natureza relacionadas a membros inferiores, ou com histórico prévio de queda. O estudo foi aprovado pelo Comitê de Ética em Pesquisa da UNIOESTE pelo parecer 111/2013 e todas as voluntárias assinaram o termo de consentimento livre e esclarecido para a participação no estudo. 
As participantes foram divididas em dois grupos, um com ausência de preocupação com quedas (GAP) e o outro com presença de preocupação com quedas (GPQ). A ferramenta utilizada para a diferenciação dos grupos foi a escala Falls Efficacy Scale International (FES-I), traduzida para o português, ${ }^{19}$ a qual é utilizada para medir o medo de quedas em 16 atividades diárias, incluindo subir e descer escadas, limpar a casa, vestirse, preparar refeições, tomar banho e sair para eventos sociais. Nessa escala o idoso é questionado sobre o quanto se sente preocupado com a possibilidade de cair ao realizar um tipo específico de atividade, representada com gestos específicos no questionário. A pessoa pode escolher entre quatro alternativas, com pontuação de um a quatro, sendo que a pontuação total pode variar de 16 a 64 . Os valores $16-25$ referem-se à ausência de preocupação com quedas; os valores 26-40 referem-se à preocupação com quedas; e os valores 41-64 referem-se à preocupação extrema com quedas durante as atividades mostradas. ${ }^{19}$

Todas as voluntárias que preencheram os critérios de inclusão responderam à escala, aplicada por um avaliador previamente treinado, que entrevistou uma voluntária de cada vez em uma sala disponibilizada pelo CRF da UNIOESTE e conhecida pelas participantes, para que se evitasse um ambiente hostil e desconfortável, que pudesse de alguma forma comprometer a fidedignidade dos resultados. Com os escores estabelecidos pela FES-I formaram-se os grupos GAP e GPQ. Foram inseridas no GAP apenas as participantes com escores entre 16 e 25, e no GPQ foram incluídas as participantes com escore acima de 25 .

\section{Avaliação funcional: teste Timed Up and Go}

O teste funcional escolhido para a avaliação da mobilidade das idosas com ou sem preocupação com quedas foi o TUG. Valores $\geq 13,5$ segundos indicam alta probabilidade de quedas e valores $<13,5$ segundos indicam idosos não caidores. ${ }^{14}$ Esse teste foi desenvolvido na tentativa de quantificar o desempenho da mobilidade, através da velocidade do idoso ao realizar uma tarefa. Dessa forma, vem sendo frequentemente utilizado para examinar a mobilidade funcional em idosos. ${ }^{20,21}$

O teste TUG foi realizado de acordo com Podsiadlo e Richardson. ${ }^{21}$ Com o voluntário sentado em uma cadeira, o avaliador opera o comando verbal para que o mesmo se levante sem ajuda dos braços, ande 3 metros (demarcados), vire, caminhe de volta os 3 metros e sente novamente na cadeira. $\mathrm{Na}$ fase inicial do teste, as idosas deveriam estar com a região dorsal apoiada ao encosto da cadeira e, ao final, deveriam voltar à posição original. $\mathrm{O}$ avaliador cronometrava o tempo do percurso, sendo que o cronômetro utilizado foi o modelo PH-CD da marca BIO (Florianópolis, Brasil). $O$ teste foi realizado uma vez para familiarização e uma segunda vez para tomada do tempo. No presente estudo um avaliador cego (ou seja, que desconhecia o grupo em que o participante havia sido alocado) executou o teste em todos os participantes de ambos os grupos.

\section{Avaliação cinética: descida de escada}

Para a realização do estudo foi construída uma escada instrumentada que possibilitou a execução do gesto de descida o mais próximo possível do cotidiano. A elaboração da escada atendeu às recomendações de Yu et al. ${ }^{22}$ Foi confeccionada em base de madeira, com sete degraus de $18 \mathrm{~cm}$ de altura, $28 \mathrm{~cm}$ de profundidade e com largura de $1 \mathrm{~m}$, com um corrimão para propiciar segurança; uma plataforma de força de $46 \mathrm{~cm} \times 49 \mathrm{~cm}$ (OR6-6 AMTI, Estados Unidos) foi camuflada por um tapete de borracha preto e alocada no quarto degrau da escada. Antes do primeiro degrau e após o último, havia uma passarela com $2 \mathrm{~m}$ de comprimento para que as voluntárias iniciassem e terminassem o movimento com uma breve caminhada. Essas dimensões obedeceram às normas propostas pela Associação Brasileira de Normas Técnicas para a construção de escadas (NBR 9077/1993). A plataforma de força foi utilizada para aquisição dos dados cinéticos de forças de reação do solo. Os sinais da plataforma de força foram capturados em um módulo da própria plataforma por meio do software AMTI Netforce, com frequência de amostragem de $100 \mathrm{~Hz}$.

A escada estava situada nas dependências do Laboratório de Pesquisa do Movimento Humano (LAPEMH) da UNIOESTE, local diverso daqueles utilizados para aplicação do questionário e realização do TUG. O LAPEMH é constituído por uma sala ampla para coleta de dados, com as paredes e o teto de coloração preta. As voluntárias executaram a descida de escada com roupas de ginástica e sem calçados. Elas foram previamente orientadas a treinar a atividade durante cinco vezes, para familiarização do procedimento e para que durante a descida atingissem a plataforma com a perna dominante, definida pela aplicação do inventário de Waterloo. ${ }^{22}$ Após o treinamento, com as voluntárias confiantes sobre a atividade que deveriam desempenhar, foi iniciada a coleta de dados, novamente realizada por um avaliador cego quanto aos grupos. 
Estando no local mais alto da escada, as participantes executaram uma pequena caminhada até chegar ao primeiro degrau e, a partir daí, começaram a descer de forma contínua, com velocidade e ritmo autosselecionados, de maneira a assemelhar-se com a atividade normal do dia-a-dia. Ao chegar ao último degrau, continuaram o movimento com uma pequena caminhada no solo por mais $2 \mathrm{~m}$. O movimento de descida foi executado até que se obtivessem cinco gestos completos. Para as análises foi considerada a média das cinco tentativas.

A plataforma de força permitiu a determinação da força vertical de reação do solo $(\mathrm{Fz})$ e a variável utilizada no presente estudo foi o primeiro pico da Fz. A nomenclatura da força vertical é controversa, sendo que em algumas referências a mesma é referida como Fy. Embora a coordenada cartesiana " $y$ " seja a vertical, na ciência espacial as forças cabeça-pé se referem ao eixo $\mathrm{Z}$, enquanto as látero-laterais ao $\mathrm{Y}$ e a ântero-posterior ao X. Esta é a nomenclatura utilizada neste estudo e em outras fontes importantes em análises cinéticas. ${ }^{17}$

Os dados provenientes da plataforma de força foram processados utilizando os softwares AMTI Bioanalysis $^{\circledR}$ (AMTI, Estados Unidos). Os sinais foram filtrados digitalmente com filtro tipo Butterworth de ordem 4 e passa baixa de $5 \mathrm{~Hz}$ e foram, em seguida, normalizados pela massa do indivíduo. ${ }^{23}$

\section{Análise estatística}

Para a análise estatística, primeiramente fez-se a análise descritiva dos dados em média e desvio padrão. A normalidade foi testada e confirmada pelo teste Shapiro-Wilk. Para comparações do primeiro pico da $\mathrm{Fz}$ e do TUG, foi utilizado o teste $\mathrm{t}$ de Student para amostras independentes.

\section{RESULTADOS}

A amostra do estudo foi composta por 31 idosas, sendo 17 delas incluídas no GAP e 16 incluídas no GPQ. No GAP o peso foi $66,1 \pm 6,33 \mathrm{~kg}$, a altura foi $1,59 \pm 0,03$ $\mathrm{m}$ e a idade foi $66,57 \pm 4,1$ anos. No GPQ o peso foi $68,88 \pm 10,38 \mathrm{~kg}$, a altura foi $1,59 \pm 0,04 \mathrm{~m}$ e a idade foi $66,67 \pm 5,8$ anos, não havendo diferença estatística entre os grupos para os dados antropométricos $(p=0,123$ e $\mathrm{p}=0,745$ respectivamente para peso e altura) e idade $(\mathrm{p}=0,901)$.

No TUG, o GAP desempenhou o teste em 9,71 $\pm 1,02$ segundos, de forma mais veloz em relação ao GPQ, que realizou o mesmo em 11,5 $\pm 1,04$ segundos $(\mathrm{p}=0,008)$ (Figura 1).
Analisando o primeiro pico da $\mathrm{Fz}$ na descida de escada, não se encontraram diferenças entre os grupos: no GPQ o valor foi $628 \pm 75$ Newtons, representando $1,17 \pm 0,18 \%$ do peso corporal; no GAP o valor foi $613 \pm 52$ Newtons, representando $1,13 \pm 0,04 \%$ do peso corporal $(\mathrm{p}=0,66)$ (Figura 2).

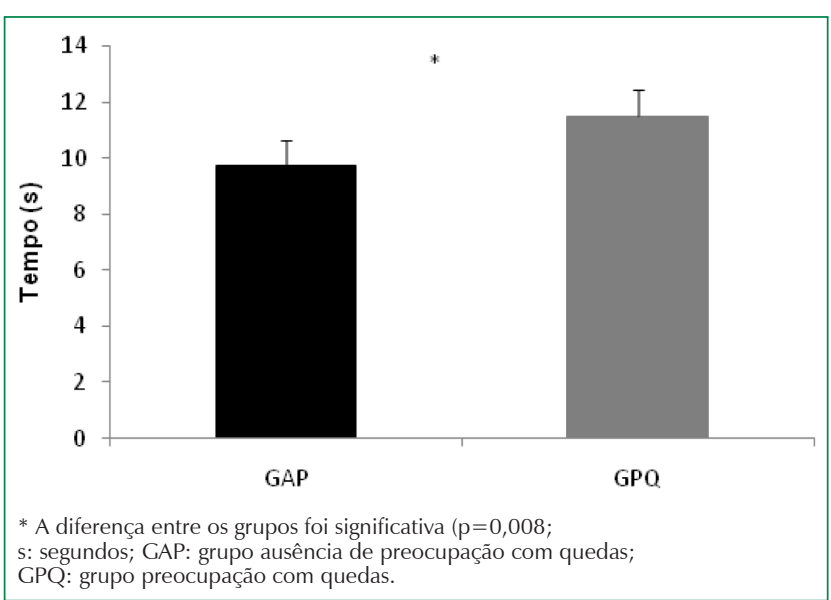

Figura 1. As barras largas representam o tempo que as idosas levaram para desempenhar o teste Timed Up and Go e as barras finas representam o desvio padrão da amostra

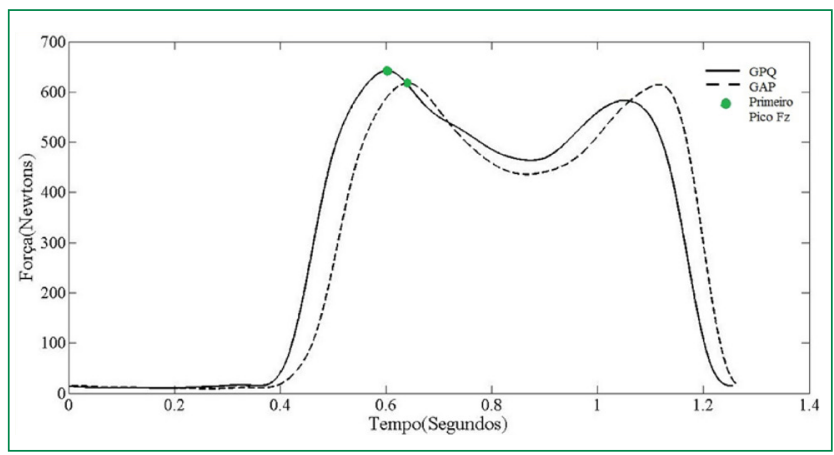

Figura 2. Curva média da força vertical de reação do solo dos grupos GPQ (Grupo preocupação com quedas) e GAP (Grupo ausência de preocupação com quedas), durante descida de escada. Não houve diferença estatística significativa entre os primeiros picos da força vertical de reação do solo $(F z)(p=0,66)$.

\section{DISCUSSÃO}

A literatura mostra que, apesar de a Fz apresentar dois picos, tanto na marcha em terreno plano como na descida de escadas, nesta última o primeiro pico vertical é maior e o segundo pico é menor em relação à marcha em terreno plano,,$^{15}$ however, there are no reports on slip resistance requirements for stair descent. The aim of this study was to determine the required 
coefficient of friction (RCOF o que concorda com o verificado no presente estudo. Isso demonstra que as atividades de deslocamento no plano e em degraus são tarefas distintas e com demandas diferentes para o corpo. Além disso, já é conhecido que transpor escadas é uma tarefa consideravelmente mais exigente quando comparada à caminhada em superfície plana..$^{21,23,24}$ Portanto, no presente estudo optou-se por utilizar o gesto funcional de descida de escadas para a avaliação das idosas.

De acordo com Podsiadlo e Richardson, ${ }^{21}$ no teste TUG valores de tempo menores que 10 segundos sugerem indivíduos totalmente livres e independentes. Já sujeitos que realizam o teste entre 10 e 19 segundos são considerados relativamente independentes, pois apresentam razoável equilíbrio e velocidade de marcha. A maioria desses sujeitos caminha livremente por mais de 500 metros, sobe escadas e sai de casa sem acompanhantes. As pessoas que ultrapassam os 20 segundos, atingindo até 29 segundos, demonstram dificuldades para as tarefas da vida diária. Escores superiores a meio minuto tendem a aparecer em pessoas totalmente dependentes para muitas atividades básicas e instrumentais da vida diária. ${ }^{22}$ No presente estudo, os dois grupos tiveram média que se enquadraram entre 10 e 19 segundos. No entanto, as idosas que tinham preocupação com quedas desempenharam o TUG de forma mais lenta, quando comparadas ao grupo das que não possuíam essa preocupação, resultado este que mostra a influência da preocupação com quedas em um teste funcional. Estes resultados estão de acordo com o estudo de Dias et al., ${ }^{25}$ que notaram menor velocidade na marcha e menor nível de independência para realização das atividades de vida diária nos idosos preocupados com quedas.

As idosas incluídas neste estudo nunca haviam vivenciado episódios de queda. No entanto, nota-se que apenas a preocupação em cair as coloca em uma condição funcional de maior risco do que as idosas sem preocupação. Thanthrige et al. ${ }^{14}$ encontraram relação inversa entre aumento do risco de quedas e nível cognitivo de idosos, o que mostra que não apenas características físicas e fisiológicas do processo de envelhecimento estão envolvidas com o risco de quedas. Segundo esses autores, idosos com menor nível cognitivo tendem a ter dificuldade na criação de estratégias rápidas para resolução de problemas, devido a isso aumenta-se o o risco de quedas. ${ }^{14}$ Além disso, a preocupação com quedas pode alterar a biomecânica fisiológica da marcha, com a alteração de velocidade, deslocamento do centro de massa e outros fatores, aumentando o risco de quedas, informação que está de acordo com os resultados obtidos pelo presente estudo no teste funcional.

No presente estudo não foram encontrados valores acima de 20 segundos no teste TUG, diferente dos resultados obtidos por Bretan et al., ${ }^{9}$ no qual em 102 indivíduos testados, $16,5 \%$ executaram o teste com o tempo entre 20 e 29 segundos. A explicação para esta divergência dos resultados está no critério adotado pelo referido estudo, que não excluiu de sua amostra idosas com episódios prévios de quedas. Esse fato pode ter contribuído para que aqueles autores encontrassem sujeitos desempenhando o teste de forma mais lenta. ${ }^{9,20}$

A baixa autoeficácia encontrada em idosos ao subir e descer escadas, em estudos que os comparam a jovens, tem sido relacionada com frequência à falta de confiança sobre as capacidades motoras, além da presença de déficits motores e mecânicos. ${ }^{1,25-27}$ A hipótese do presente estudo foi que idosas com preocupação em cair teriam o primeiro pico da Fz menor com relação a idosas sem preocupação. Entretanto, os resultados encontrados negaram essa hipótese: apesar das diferenças obtidas no teste funcional, a Fz não foi estatisticamente diferente entre os grupos.

Um estudo comparou o primeiro pico da $\mathrm{Fz}$ em idosas e adultas jovens e mostrou que as idosas tiveram menores magnitudes no momento de acomodação. ${ }^{15}$ Esse achado contradiz o resultado encontrado no presente estudo, quando se compararam idosas com e sem medo de cair, pois pode-se fazer uma analogia entre idosas sem medo e adultas jovens, as quais geralmente não têm preocupação com quedas. Talvez a variável primeiro pico da $\mathrm{Fz}$ não seja sensível o suficiente para identificar diferenças entre os grupos, e esta pode ter sido uma limitação deste estudo. No entanto, Bertucco e Cesari ${ }^{28}$ realizaram uma coleta de familiarização prévia à coleta de dados. Não ter realizado uma coleta de familiarização em semanas anteriores à coleta de dados pode ter sido também uma limitação que impossibilitou as diferenças nos dados cinéticos, uma vez que o teste de descida de escada foi realizado em um ambiente desconhecido às voluntárias. Em contrapartida, no teste TUG, as idosas estavam no ambiente do centro de reabilitação física, no qual realizam atividades rotineiramente; nesse ambiente o fator desconhecido foi eliminado, podendo ter sido um dos fatores que possibilitaram aparecer a diferença entre os grupos.

Futuros estudos podem ser desenvolvidos com aplicação de intervenções multidisciplinares que enfoquem, além de aspectos motores, aspectos psicológicos de preocupação com as quedas. Poderão 
ser utilizadas, também, ferramentas biomecânicas e testes funcionais como marcadores de evolução dessas pessoas. Além de variáveis cinéticas, variáveis cinemáticas de membros inferiores, como por exemplo a flexão de joelho e quadril, têm potencial de contribuição para esse tipo de estudo.

Foram encontrados, neste estudo, resultados interessantes com relação ao desempenho de idosas com e sem preocupação em cair. É importante ressaltar a originalidade do estudo, que foi capaz de incluir uma variável biomecânica e testes clínicos em idosas com medo e sem medo de queda, informação até então inédita na literatura. Foi possível verificar menor desempenho no teste TUG no grupo de idosas com preocupação em sofrer quedas comparado ao grupo de idosas sem essa preocupação. Analisando a força vertical de reação do solo, durante o movimento de descida de escadas, não foram encontradas diferenças durante o contato inicial da fase de apoio, entre os dois grupos avaliados.

\section{REFERÊNCIAS}

1. Mello PMS, Silva AA, Gonçalves PP, Santos NLR, Toledano M, Mendes PW, Maciel MHC, Franzoi AC. Tradução, adaptação cultural e validação de uma escala para aferir limitação da atividade de subir e descer escadas. Rev Bras Geriatr Gerontol. 2013 Sept;16(3):433-41.

2. Lopes KT, Costa DF, Santos LF, Castro DP, Bastone AC. Prevalência do medo de cair em uma população de idosos da comunidade e sua correlação com mobilidade , equilíbrio dinâmico , risco e histórico de quedas. Rev Bras Fisioter. 2009;13(3):223-9.

3. Nunes BP, de Oliveira Saes M, Siqueira FV, Tomasi E, Silva SM, da Silveira DS, Soares MU, Facchini LA, Thumé E. Falls and selfassessment of eyesight among elderly people: a population-based study in a south Brazilian municipality. Arch Gerontol Geriatr. 2014 July-Aug;59(1):131-5.

4. Lee CW, Cho GH. Effect of stationary cycle exercise on gait and balance of elderly women. J Phys Ther Sci. 2014 Mar;26(3):431-3.

5. Startzell JK, Owens DA, Mulfinger LM, Cavanagh PR. Stair negotiation in older people: a review. J Am Geriatr Soc. 2000 May; 48(5):567-80.

6. Nardi EDFR, Oliveira MLF. Significado de cuidar de idosos dependentes na perspectiva do cuidador familiar. Cienc Cuid Saúde. 2009 Dez;8(3):428-35.

7. Sheehan RC, Gottschall JS. At similar angles, slope walking has a greater fall risk than stair walking. Appl Ergon. 2012 May;43(3):473-8.

8. Tiedemann AC, Sherrington C, Lord SR. Physical and psychological factors associated with stair negotiation performance in older people. J Gerontol A Biol Sci Med Sci. 2007 Nov;62(11):1259-65.

9. Bretan O, Silva Júnior JE, Ribeiro OR, Corrente JE. Risk of falling among elderly persons living in the community: assessment by the Timed up and go test. Braz J Otorhinolaryngol. 2013 Jan-Feb;79(1):18-21.

10. Carli JVM, Bohrer RCD, Lodovico A, Rodacki ALF. Kinetic analysis of floor-to-stair transition gait of elderly with different functional levels. Rev Bras Cineantropom Desempenho Hum. 2014;16(1):66-75.

11. Legters K. Fear of falling. Phys Ther. 2002 Mar;82(3):264-72.

12. Chantanachai T, Pichaiyongwongdee S, Jalayondeja C. Fall prediction in thai elderly with timed up and go and tandem walk test: a crosssectional study. J Med Assoc Thai. 2014 Jul;97 Supp1 7:S21-5.

13. Soto-Varela A, Faraldo-García A, Rossi-Izquierdo M, Lirola-Delgado A, Vaamonde-Sánchez-Andrade I, Del-Río-Valeiras M, GayosoDiz P, Santos-Pérez S. Can we predict the risk of falls in elderly patients with instability? Auris Nasus Larynx. 2014 Sept 3. pii: S03858146(14)00117-5.

14. Thanthrige RS, Dassanayake S, Dissanayake D. Relationship between increased risk of falling and cognitive impairment in residents of an elderly home in the Colombo district. Ceylon Med J. 2014 Mar;59(1):21-3.

15. Christina KA, Cavanagh PR. Ground reaction forces and frictional demands during stair descent: effects of age and illumination. Gait Posture. $2002 \mathrm{Apr} ; 15(2): 153-8$.

16. Lee HJ, Chou LS. Balance control during stair negotiation in older adults. J Biomech. 2007;40(11):2530-6.

17. Zatsiorsky VM. Kinetics of human motion. Champaign: Human Kinetics; 2002.

18. Milner CE, Hamill J, Davis I. Are knee mechanics during early stance related to tibial stress fracture in runners? Clin Biomech (Bristol, Avon). 2007 July;22(6):697-703.

19. Camargos FFO, Dias RC, Dias JMD, Freire MTF. Adaptação transcultural e avaliação das propriedades psicométricas da Falls Efficacy Scale - International em idosos brasileiros (FES-I-BRASIL). Rev Bras Fisioter. 2010;14(3):237-43.

20. Gervais T, Burling N, Krull J, Lugg C, Lung M, Straus S, Jaglal S, Sibley KM. Understanding approaches to balance assessment in physical therapy practice for elderly inpatients of a rehabilitation hospital. Physiother Can. 2014 Winter;66(1):6-14.

21. Podsiadlo D, Richardson S. The timed "Up \& Go": a test of basic functional mobility for frail elderly persons. J Am Geriatr Soc. 1991 Feb;39(2):142-8.

22. Yu B, Kienbacher T, Growney ES, Johnson ME, An KN. Reproducibility of the kinematics and kinetics of the lower extremity during normal stair-climbing. J Orthop Res. 1997 May;15(3):348-52. 
23. Winter D. Biomechanics and motor control of human movement. 4th ed. Hoboken: John Wiley \& Sons; 2009.

24. Hardt J, Benjanuvatra N, Blanksby B. Do footedness and strength asymmetry relate to the dominant stance in swimming track start? J Sports Sci. 2009 Sept;27(11):1221-7.

25. Dias RC, Freire MTF, Santos ÉGS, Vieira RA, Dias JMD, Perracini MR. Características associadas à restrição de atividades por medo de cair em idosos comunitários. Rev Bras Fisioter. 2011;15(5):406-13.

26. Paquette MR, Zhang S, Milner CE, Fairbrother JT, Reinbolt JA. Effects of increased step width on frontal plane knee biomechanics in healthy older adults during stair descent. Knee. 2014 Aug;21(4):821-6.

27. Buckley JG, Cooper G, Maganaris CN, Reeves ND. Is stair descent in the elderly associated with periods of high centre of mass downward accelerations? Exp Gerontol. 2013 Feb;48(2):283-9.

28. Bertucco M, Cesari P. Dimensional analysis and ground reaction forces for stair climbing: effects of age and task difficulty. Gait Posture. 2009 Feb;29(2):326-31. 